\title{
Effects of ipsilateral cerebellum ablation on acquisition and retention of classically conditioned eyeblink responses in rats
}

Takahiro Horiuchi ${ }^{\mathrm{a}}$, Shigenori Kawahara ${ }^{\mathrm{b}, *}$

${ }^{a}$ Graduate School of Pharmaceutical Sciences, The University of Tokyo, 7-3-1 Hongo, Bunkyo-ku, Tokyo 113-0033, Japan

${ }^{\mathrm{b}}$ Graduate School of Science and Engineering, University of Toyama, 3190 Gofuku, Toyama 930-8555, Japan

This manuscript contains 12 text pages, 3 figures, and no tables.

*Correspondence should be addressed to Dr. Shigenori Kawahara, Graduate School of Science and Engineering, University of Toyama, 3190 Gofuku, Toyama 930-8555, Japan. (Tel.: +81 76445 6871; Fax: +81 76445 6697; E-mail address: kawahara@eng.u-toyama.ac.jp)

Acknowledgements: We thank Dr. Yutaka Kirino and Dr. Norio Matsuki for their helpful suggestions. This work was supported by grants from the Ministry of Education, Culture, Sports, Science and Technology of Japan (\#21570161) and from Takeda Science Foundation.

Keywords: Eyeblink conditioning, Cerebellum, Learning, Memory 


\begin{abstract}
The ipsilateral cerebellum to the trained eye has been reported to be essential for acquisition and retention of the conditioned response (CR) in rabbit eyeblink conditioning. Although pharmacological studies have suggested its important roles in other species too, to what degree does eyeblink conditioning in rats depend on the ipsilateral cerebellum is not clear. In this work, we ablated the ipsilateral cerebellum in rats before or after conditioning to examine its roles in acquisition and retention of the CR. In the first experiment, rats received ablation of the ipsilateral cerebellum and recovered for more than 3 weeks. They then underwent eyeblink conditioning for 7 days with a tone and a periorbital electrical shock. Consistent with other previous reports, hemicerebellectomized rats showed significant impairment compared to sham-lesioned rats. However, the hemicerebellectomized rats acquired CRs to some degree, and the acquired CR showed adaptive timing. In the second experiment, rats received the hemicerebellectomy after acquiring CR by 7 days of conditioning in a delay paradigm. After more than 3 weeks of recovery, they were again conditioned in a delay paradigm. Rats with ipsilateral cerebellar lesions showed severe impairment in retention of the pre-acquired $\mathrm{CR}$; however, they reacquired $\mathrm{CR}$ to some degree during the subsequent reconditioning sessions. These results suggest that the ipsilateral cerebellum plays an important role in rat eyeblink conditioning as well but that other brain regions can partially compensate for its removal.
\end{abstract}


Classical eyeblink conditioning is one of the most extensively studied models for associative learning $[1,2,3,4]$. In this task, a corneal air puff or periorbital shock (unconditioned stimulus, US) is delivered to the eye to elicit a reflexive blink (unconditioned response). When a preceding neutral conditioned stimulus (CS) such as a tone is repeatedly paired with a US, animals blink in response to a CS. This is known as a conditioned response (CR), and it occurs in a carefully timed manner before a US. Cumulative evidence has clearly shown that the cerebellum and brain stem play a critical role in acquisition and retention of the $\mathrm{CR}$ in delay eyeblink conditioning in rabbits. On the basis of the results from lesioning [5] or reversible inactivation [6] of the deep cerebellar nuclei, the ipsilateral cerebellum to the trained eye was concluded to be essential for CR acquisition, retention, and expression. Similarly, CR acquisition and retention in rats were impaired after permanent lesions of the bilateral cerebellar nucleus $[7,8]$ or reversible inactivation of the ipsilateral cerebellum [9]. In addition, infant rats that received unilateral lesions of the ipsilateral cerebellum on postnatal day 10 or 20 were unable to acquire CR during 3-day conditioning [10]. Although one of the previous studies reported personal observations that large unilateral cerebellar nuclei lesions did not diminish pre-acquired CR in some rats [7], other studies suggested that the ipsilateral cerebellum might be essential for CR acquisition and retention in rats in the same way it is in rabbits $[9,10]$.

In the present study, we investigated the effects of ipsilateral cerebellum ablation on acquisition and retention of CR in delay eyeblink conditioning in adult rats. Sixty-one 10-week-old male Wistar rats (Japan SLC, Inc., Hamamatsu, Shizuoka, Japan) were used. The animals were housed in standard plastic cages with free access to food and water in a colony room with a 12-h light/dark cycle. All of the experimental procedures were performed during the light period of the cycle in accordance with the guidelines established by the institutional animal investigation committee at the University of Tokyo and the NIH Guide for the Care and Use of Laboratory Animals. Efforts were made to optimize the comfort as well as minimize the use of the animals.

We performed 2 types of lesion experiments: pre-conditioning lesions for testing learning ability, and post-conditioning lesions for testing memory retention. In the pre-conditioning lesion experiment, rats were randomly assigned to receive either ipsilateral cerebellar lesion (CBL group) or sham surgery (sham lesion group). Rats were anesthetized with sodium pentobarbital (65 mg/kg i.p., Kyoritsu Seiyaku, Tokyo, Japan). Isofluran (1-2\%, Abbot Japan, Osaka, Japan) was also used when necessary. After removal of the overlying skull and dura, the ipsilateral cerebellum was ablated by aspiration to the level of the deep 
cerebellar nuclei. The resulting cavity was then filled with sterile gel foam (Pfizer Japan Inc, Tokyo, Japan) and the wound edges were sutured. The animals were then injected with ampicillin (100 mg/kg i.p., Meiji Seika, Tokyo, Japan) and warmed until they moved spontaneously. The sham lesion group received the craniotomy but not the cerebellar ablation. After 3 weeks of recovery, 4 Teflon-coated stainless-steel wires (\#7910; A-M Systems, Carlsborg, WA, USA) were subcutaneously implanted in the left upper eyelid under anesthesia with sodium pentobarbital $(65 \mathrm{mg} / \mathrm{kg})$. These wires were soldered to the pins of a connector that was secured to the skull with dental acrylic resin and stainless-steel screws. Two to 4 days after surgery, spontaneous eyeblink frequency was measured for 2 days and then the rats were conditioned for 7 days. A daily session consisted of 100 trials grouped into 10 blocks, each of which included 9 CS-US paired trials followed by 1 CS-alone trial. Trials were separated by a variable inter-trial interval pseudo-randomized between 20 and $40 \mathrm{~s}$ with a mean of $30 \mathrm{~s}$. The CS was a 350-ms tone $(5 \mathrm{kHz}, 85-90 \mathrm{~dB})$ with a rise/fall time of $10 \mathrm{~ms}$. US consisted of a $100-\mathrm{ms}$ periorbital shock $(1.5 \mathrm{~mA}, 100-\mathrm{Hz}$ square pulses $)$ that was delivered through a pair of electrodes implanted in the left upper eyelid. CR was monitored through electromyographic (EMG) activity recorded at a sampling rate of $10 \mathrm{kHz}$ with another pair of implanted electrodes. Rats in the CBL and sham lesion groups received either paired conditioning or pseudoconditioning. In paired conditioning (CBL: $\mathrm{n}=11$; sham: $\mathrm{n}=7$ ), the CS preceded and co-terminated with the US. In pseudo-conditioning (CBL: $n=7$, sham: $n=9$ ), a pseudo-randomized stimulus-free time (between 6 and 20 s) was interposed between the CS and the US.

In the post-conditioning lesion experiment, rats first received the surgery for implanting the wire electrodes as described above. After 2 to 4 days, spontaneous eyeblinks were recorded and then the rats were conditioned for 7 days in the paired conditioning paradigm. The rats whose mean percentage of CRcontaining trials (CR\%) during the last 2 days of acquisition sessions exceeded $70 \%$ were divided into 2 groups and received either sham surgery (sham, $\mathrm{n}=12)$ or ipsilateral cerebellum ablation $(\mathrm{CBL}, \mathrm{n}=15)$ on the next day of the last acquisition session. At least 3 weeks after the surgery, the rats were conditioned again for 10 days to test their memory retention and reacquisition.

The EMG data were analyzed as described previously [11]. Briefly, mean + SD of the amplitudes of the EMG signal for $300 \mathrm{~ms}$ before the CS in 100 trials was defined as the threshold and was used in the following analysis. In each trial, average EMG amplitudes exceeding the threshold were calculated for 300 ms before CS onset (pre-value) and for $100 \mathrm{~ms}$ before US onset (CR value). If the pre-value was less than 
$10 \%$ of the threshold, the trial was regarded as a valid trial. Among the valid trials, a trial was assumed to contain $\mathrm{CR}$ if the $\mathrm{CR}$ value exceeded 10 times the pre-value. In CS-alone trials, the period for CR value calculation was extended by $100 \mathrm{~ms}$ to the end of the expected US. The percentage of CR-containing trials in the valid trials $(\mathrm{CR} \%)$ was expressed as mean \pm SEM. In the post-conditioning lesion experiment, we also calculated the retention ratio by dividing the $\mathrm{CR} \%$ in the first reconditioning session by that in the last acquisition session. To show the temporal CR pattern, the EMG amplitude data of each rat were averaged over the valid trials for each day. These trial-averaged EMG amplitude data were normalized by the timeaveraged values for $300 \mathrm{~ms}$ before the CS onset. CR onset latency was defined as the latency from the CS onset to the time when the EMG amplitude exceeded 10 times the pre-value for the first time. CR peak latency was defined as latency from the CS onset to the time of maximum EMG amplitude between $50 \mathrm{~ms}$ after CS and US onset. These latencies were calculated in all trials that were judged to contain CR.

After all of the behavioral experiments were completed, the rats were intraperitoneally injected with an excess amount of sodium pentobarbital $(130 \mathrm{mg} / \mathrm{kg})$ and perfused intracardially with $0.9 \%$ saline followed by phosphate-buffered $10 \%$ formalin. The brain was removed from the skull and stored in $10 \%$ formalin for a few days. After infiltration with $30 \%$ sucrose, the brain was frozen, sectioned at $60 \mu \mathrm{m}$, and stained with cresyl violet. The largest and smallest lesioned areas were reconstructed from the sections according to the stereotaxic atlas of the rat brain [12].

Statistical significance was determined by a one-way or two-way analysis of variance (ANOVA) with repeated measures or by a $t$-test using the SPSS statistical software (SPSS Japan, Tokyo, Japan). We also used the Tukey HSD test for post hoc analysis. A value of $P<0.05$ was regarded as significant.

Figure 1 summarizes the extent of the cerebellar lesions in the CBL group. In most rats, the lesioned site included both the ipsilateral cerebellar cortex and the deep cerebellar nuclei, whereas the underlying brain stem was left intact. In some rats, a large part of the vermis was also ablated. Rats whose contralateral medial and interpositus nuclei were lesioned were excluded from subsequent analyses.

In the pre-conditioning lesion experiment, the CBL group showed severe impairment during delay eyeblink conditioning, though they learned to some degree, while the sham lesion group readily acquired CR (Fig. 2A). The pseudo-conditioned CBL and sham lesion groups did not learn at all. Two-way repeated measures ANOVA revealed significant group effects $\left(\mathrm{F}_{(3,30)}=51.31, P<0.01\right)$, session effects $\left(\mathrm{F}_{(6,180)}=\right.$ 22.05, $P<0.01)$, and interaction between groups and sessions $\left(\mathrm{F}_{(18,180)}=5.98, P<0.01\right)$. Post hoc Tukey 
HSD tests indicated that there were significant differences between the pair- and the pseudo-conditioned groups $(P<0.01)$ and between the pair-conditioned $\mathrm{CBL}$ and the pair-conditioned sham lesion groups $(P=$ 0.01). One-way repeated measures ANOVAs also confirmed that the CR\% of the pair-conditioned CBL and sham lesion groups significantly increased during conditioning $\left(\mathrm{CBL}: \mathrm{F}_{(6,60)}=8.07, P<0.01\right.$, sham: $\mathrm{F}_{(6,36)}$ $=14.30, P<0.01)$ and that of the pseudo-conditioned groups did not. To investigate the temporal pattern of the CR acquired by the CBL group, we calculated normalized EMG amplitude in the last session and compared it with that acquired by the pair-conditioned sham lesion group (Fig. 2B). The normalized EMG amplitude data of the lower CR\% of the pair-conditioned CBL group was smaller than that of the pairedconditioned sham lesion group. However, the temporal pattern was similar between them. Quantitative analysis of the latencies to the CR onset and the peak confirmed that there were no significant differences between the CBL group and the sham lesion group in the temporal CR pattern (Fig. 2C; onset latency: $P=$ 0.43 by $t$-test; peak latency: $P=0.23$ by $t$-test). These results suggest that ipsilateral cerebellar lesion severely impairs CR acquisition but does not completely prevent an animal from acquiring CR which shows adaptive timing.

We next examined whether rats could retain acquired CR after cerebellum ablation. Rats received ipsilateral cerebellum ablation after learning the delay paradigm and then were conditioned again to test memory retention and relearning ability (Fig. 3A). A two-way repeated measures ANOVA confirmed that there were no significant differences between the sham lesion and CBL groups during acquisition sessions (group: $\mathrm{F}_{(1,25)}=1.15, P=0.30$; session: $\mathrm{F}_{(6,150)}=95.93, P<0.01$; session $\mathrm{x}$ group: $\mathrm{F}_{(9,150)}=0.79, P=0.53$ ). However, after cerebellum ablation, the CBL group showed a remarkable reduction in memory retention, whereas the sham lesion group showed little reduction from the last acquisition session. There was no significant difference in CR\% between the first acquisition session and the first retention session in the CBL group ( $P=0.30$ by $t$-test). Fig. 3B compares the CR\% ratio in the first retention session to that in the last acquisition session in each rat. A significant difference in retention ratio was seen between the sham lesion and $\mathrm{CBL}$ groups $(P<0.01$ by $t$-test), indicating that ipsilateral cerebellum ablation significantly impaired retention of pre-acquired $\mathrm{CR}$ in delay eyeblink conditioning. During the subsequent reconditioning sessions, the CBL group reacquired $\mathrm{CR}$ in the absence of the ipsilateral cerebellum $\left(\mathrm{F}_{(9,126)}\right.$ $=6.31, P=0.01$ by one-way repeated measures ANOVA) as in the pre-conditioning lesion experiment. However, the CR\% of the CBL group did not reach that of the sham lesion group even after 10 days of 
reconditioning ( $P=0.04$ by $t$-test). In addition, the temporal pattern of the CR in the CBL group was similar to that of the sham lesion group in the first (Fig. 3E) and the last (Fig. 3F) reconditioning sessions, although the amplitude of the CBL group was smaller than that of the sham lesion group, which corresponds to the lower post-ablation CR\%. Quantitative analysis on the latencies to the CR onset and peak in the last reconditioning session confirmed that there were no significant differences in the temporal pattern of the reacquired CR between the sham lesion and CBL groups (Fig. 3C; onset latency: $P=0.71$ by $t$-test; peak latency: $P=0.47$ by $t$-test). These results suggested that ablation of the ipsilateral cerebellum severely impairs retention of pre-acquired CR but does not completely prevent an animal from reacquiring CR with an adaptive timing.

Although rats could acquire CR to some degree in the absence of the ipsilateral cerebellum (Fig. 2), intact rats may still use the ipsilateral cerebellum to learn during eyeblink conditioning. In fact, the postconditioning lesion experiment revealed that ablation of the ipsilateral cerebellum reduced CR expression to almost the same level as in the first acquisition session (Fig. 3). This dependency on the ipsilateral cerebellum in rats is consistent with those found in other previous reports in rabbits [5], rats [9,10], and humans [13]. The apparent difference from rabbits and humans might be because of the different US in rats (periorbital electrical shock) and in other species (air puff). The difference from the previous study using rats [10] could be due to the fact that the rats were considerably younger (postnatal 10 or 20 days at the surgery) in the previous study and that they were only conditioned for three 100-trial sessions, which is less than the 7 sessions used in the present study. In rats, cerebellar lesions at an early age cause more motor deficits than at a later age (for reviews: [14]).

In addition to the critical involvement of the ipsilateral cerebellum, other brain regions are suggested to be involved in compensatory CR acquisition when the ipsilateral cerebellum was removed. Then, where is the other brain region? One of the candidates is the remaining contralateral cerebellum. A metabolic study in rats [15] and an fMRI study in rabbits [16] suggested that the contralateral cerebellum is involved in delay eyeblink conditioning (for reviews: [17]). Contribution of the contralateral cerebellum to motor recovery after unilateral cerebellar lesions in monkeys was also reported [18]. The striatum is another candidate location in which conditioning-related activities were reported in humans [19] and rabbits [20]. Contribution of the striatal excitatory transmission to the recovery from cerebellar damage was also reported in rats [21]. Further investigation is needed to examine whether these areas are involved in 
delay eyeblink conditioning in hemicerebellectomized rats.

In summary, the ipsilateral cerebellum is crucial for successful CR acquisition and retention during delay eyeblink conditioning using tone CS and periorbital shock US in rats. However, rats can acquire the CR to some degree without the ipsilateral cerebellum, which seems to be somewhat different from the delay conditioning of the nictitating membrane responses using tone CS and air puff US in rabbits. 


\section{References}

[1] K.M. Christian, R.F. Thompson, Neural substrates of eyeblink conditioning: acquisition and retention, Learn. Mem. 10 (2003) 427-455.

[2] R.F. Thompson, In search of memory traces, Ann. Rev. Psychol. 56 (2005) 1-23.

[3] J.E. Steinmetz, Brain substrates of classical eyeblink conditioning: a highly localized but also distributed system, Behav. Brain Res. 110 (2000) 13-24.

[4] J.M. Delgado-García, A. Gruart, The role of interpositus nucleus in eyelid conditioned responses, Cerebellum 1 (2002) 289-308.

[5] D.A. McCormick, G.A. Clark, D.G. Lavond, R.F. Thompson, Initial localization of the memory trace for a basic form of learning, Proc. Natl. Acad. Sci. U S A. 79 (1982) 2731-2735.

[6] V. Bracha, M.L. Webster, N.K. Winters, K.B. Irwin, J.R. Bloedel, Effects of muscimol inactivation of the cerebellar interposed-dentate nuclear complex on the performance of the nictitating membrane response in the rabbit, Exp. Brain Res. 100 (1994) 453-468.

[7] R.W. Skelton, Bilateral cerebellar lesions disrupt conditioned eyelid responses in unrestrained rats, Behav. Neurosci. 102 (1988) 586-590.

[8] T. Lee, J.J. Kim, Differential effects of cerebellar, amygdalar, hippocampal lesions on classical eyeblink conditioning in rats, J. Neurosci. 24 (2004) 3242-3250.

[9] J.H. Freeman, H.E. Halverson, A. Poremba, Differential effects of cerebellar inactivation on eyeblink conditioned excitation and inhibition, J. Neurosci. 25 (2005) 889-895.

[10] J. H. Freeman, C.S. Carter, M.E. Stanton, Early cerebellar lesions impair eyeblink conditioning in developing rats: Differential effects of unilateral lesions on postnatal day 10 or 20, Behav. Neurosci. 109 (1995) 893-902.

[11] K. Takehara, S. Kawahara, Y. Kirino, Time-dependent reorganization of the brain components underlying memory retention in trace eyeblink conditioning, J. Neurosci. 23 (2003) 9897-9905.

[12] G. Paxinos, C. Watson, The rat brain in stereotaxic coordinates, fourth edition. Academic Press, San Diego, 1998.

[13] M. Gerwig, F.P. Kolb, D. Timmann, The involvement of the human cerebellum in eyeblink conditioning, Cerebellum 6 (2007) 38-57. 
[14] A. Gramsbergen, Neural compensation after early lesions: A clinical view of animal experiments, Neurosci Biobehav Rev. 31 (2007) 1088-1094.

[15] B. Plakke, J.H. Freeman, A. Poremba, Metabolic mapping of the rat cerebellum during delay, trace eyeblink conditioning, Neurobiol Learn Mem. 88 (2007) 11-18.

[16] M.J. Miller, N.K. Chen, L. Li, B. Tom, C. Weiss, J.F. Disterhoft, A.M. Wyrwicz, fMRI of the conscious rabbit during unilateral classical eyeblink conditioning reveals bilateral cerebellar activation, J. Neurosci. 23 (2003) 11753-11758.

[17] M. Ivarsson, P. Svensson, G. Hesslow, Contralateral cerebellar involvement in conditioned eyeblink responses, Prog. Brain Res. 124 (2000) 309-316.

[18] K. Amrani, R.W. Dykes, Y. Lamarre, Bilateral contributions to motor recovery in the monkey following lesions of the deep cerebellar nuclei., Brain Res. 740 (1996) 275-284.

[19] C.G. Logan, S.T. Grafton, Functional anatomy of human eyeblink conditioning determined with regional cerebral glucose metabolism, positron-emission tomography, Proc. Natl. Acad. Sci. U.S.A. 92 (1995) 7500-7504.

[20] I.M. White, D.P. Miller, W. White, G.L. Dike, G.V. Rebec, J.E. Steinmetz, Neuronal activity in rabbit neostriatum during classical eyelid conditioning, Exp. Brain Res. 99 (1994) 179-190.

[21] D. Centonze, S. Rossi, P. De Bartolo, V. De Chiara, F. Foti, A. Musella, G. Mataluni, S. Rossi, G. Bernardi, G. Koch, L. Petrosini, Adaptations of glutamatergic synapses in the striatum contribute to recovery from cerebellar damage, Eur. J. Neurosci. 27 (2008) 2188-2196. 


\section{Figure legends}

Fig. 1. Histological evaluation of ipsilateral cerebellum ablation. (A) Typical coronal sections stained with cresyl violet through the cerebellum and brain stem in the CBL group. (B) Line drawings of the coronal sections showing the extent of the cerebellar lesions in the CBL group. The black and gray areas indicate the smallest and largest lesions, respectively. Numbers to the left indicate stereotaxic coordinates relative to bregma [12].

Fig. 2. Effects of pre-conditioning lesions of the ipsilateral cerebellum on CR acquisition during delay eyeblink conditioning. (A) CR acquisition during paired conditioning in the sham lesion (empty circle) and CBL (filled circle) groups or during pseudo-conditioning in the sham lesion (empty triangle) and CBL (filled triangle) groups. Mean percentage of $\mathrm{CR}$ occurrence $(\mathrm{CR} \%)$ is shown for each daily session. In sessions 1-2, spontaneous eyeblinks (without CS or US) were recorded. In sessions 3-9, rats received paired or unpaired CS and US presentations. (B) Normalized EMG amplitudes of the sham lesion (thin line) and CBL (thick line) groups in session 9. Filled or empty arrowhead indicates onset of CS or US, respectively. (C) Latency from CS onset to CR onset (left) and peak (right) in the sham lesion (empty column) and CBL (filled column) groups in session 9. These latencies were calculated in the CR-containing trials, averaged in each rat, and then averaged over the rats in each group. Data are mean \pm SEM.

Fig. 3. Effects of post-conditioning lesions of the ipsilateral cerebellum on retention of pre-acquired CR and CR reacquisition during subsequent delay eyeblink conditioning. (A) CR acquisition and reacquisition before (sessions 3-9) and after (sessions 10-19) hemicerebellectomy in the sham lesion (empty circle) and CBL (filled circle) groups. More than 3 weeks were allowed for recovery between sessions 9 and 10 . Spontaneous eyeblinks (without CS or US) were recorded in sessions 1-2. (B) Retention ratio in the sham lesion (empty column) and CBL (filled column) groups calculated by dividing the CR\% in session 10 by that in session 9 in each rat. An asterisk (*) indicates $P<0.05$. (C) Latency of onset (left) and peak (right) of the reacquired CR in sham lesion (empty column) and CBL (filled column) groups in session 19. Latencies were calculated in the CR-containing trials, averaged in each rat, and then averaged over the rats in each group. (D-F) Normalized EMG amplitudes of the sham lesion (thin line) and CBL (thick line) groups in sessions 9 (D), 10 (E), and 19 (F). Filled or empty arrowhead indicates onset of CS or US, 
respectively. 
(A)

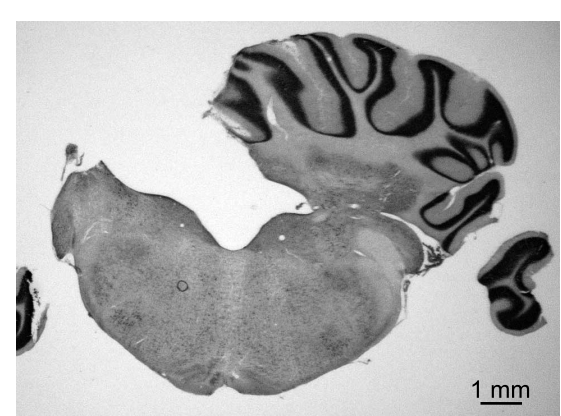

(B)

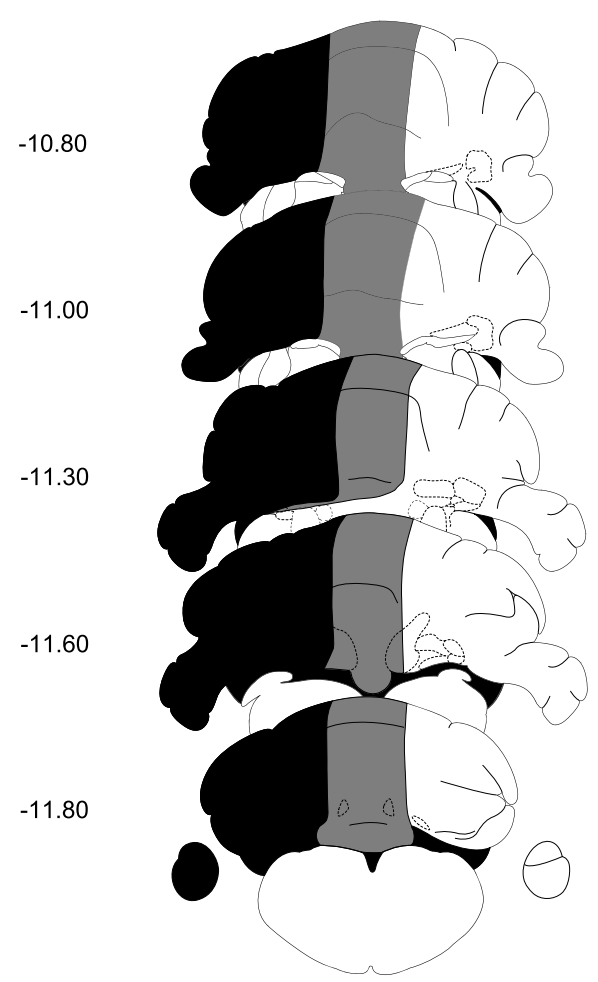


(A)

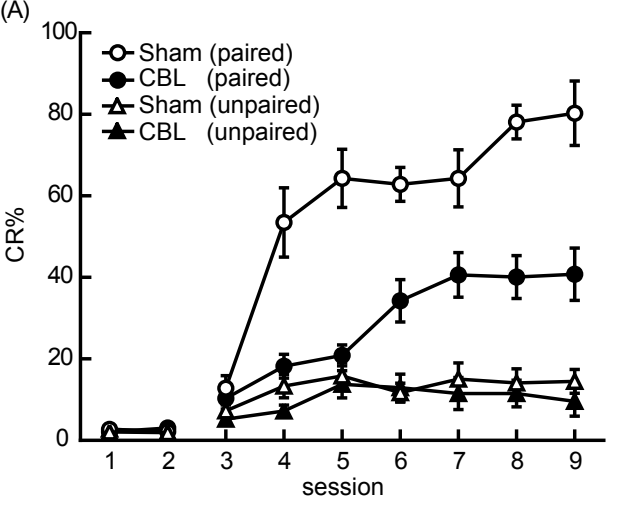

(B)

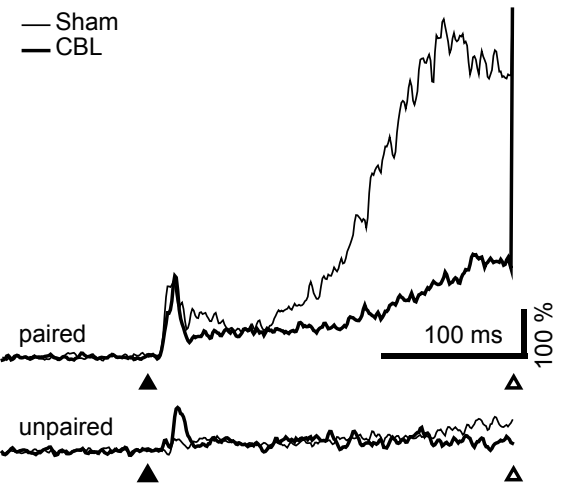

(C)

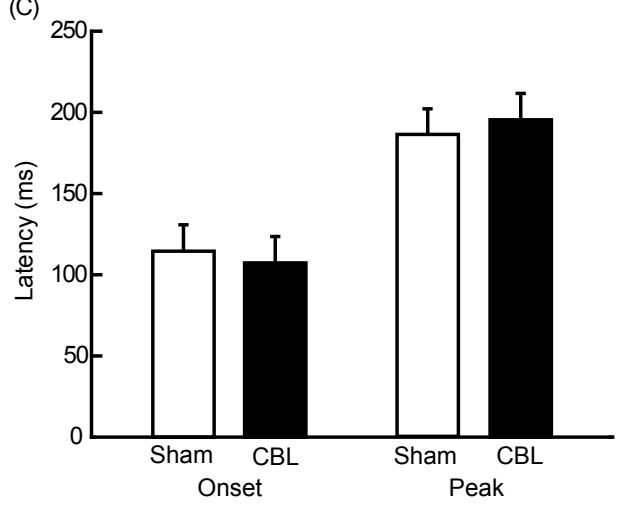



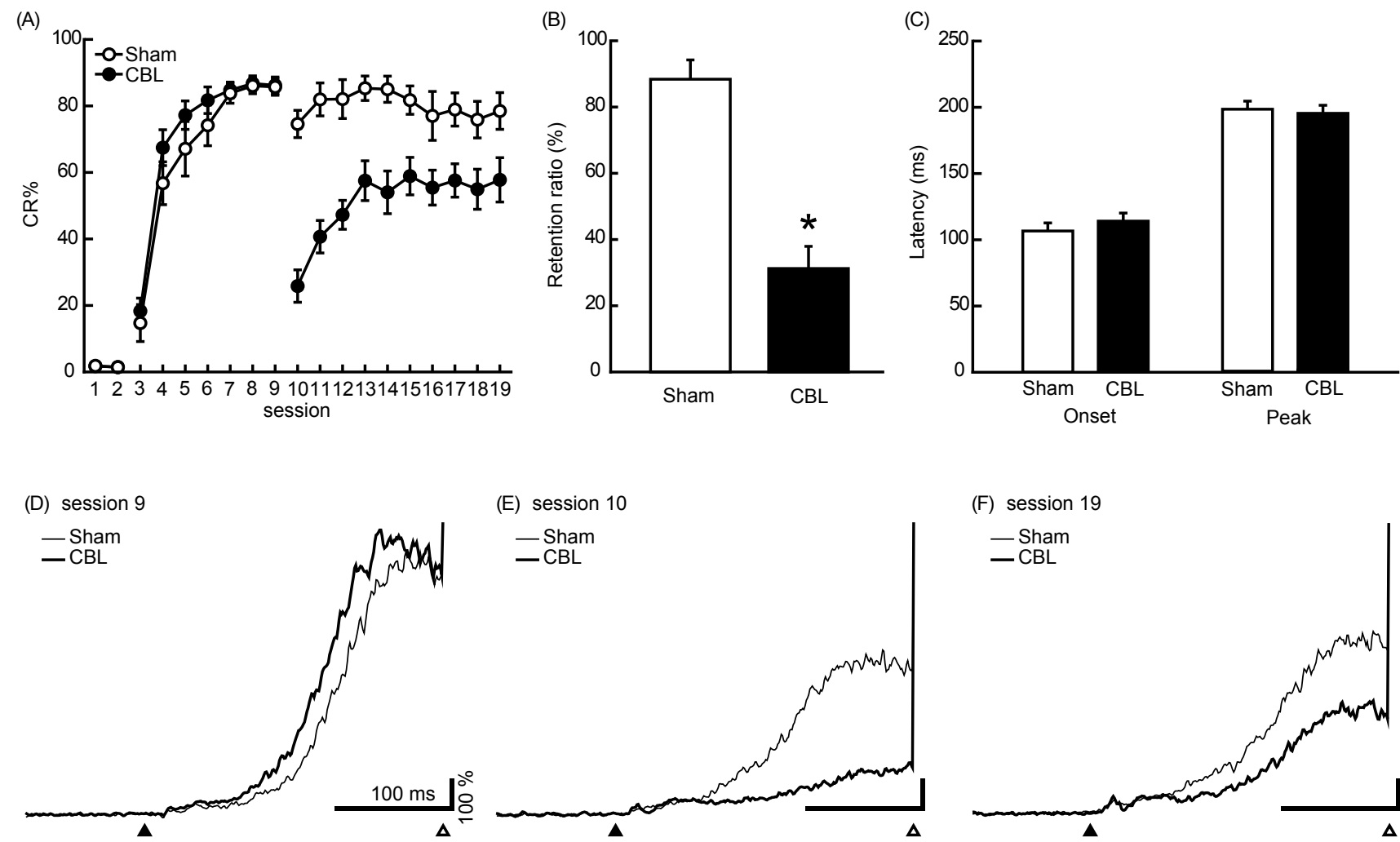

(E) session 10

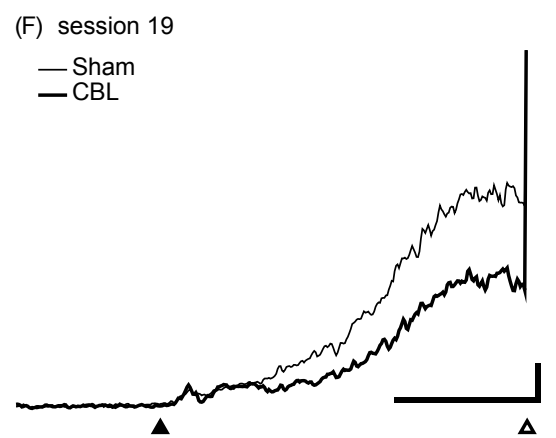

